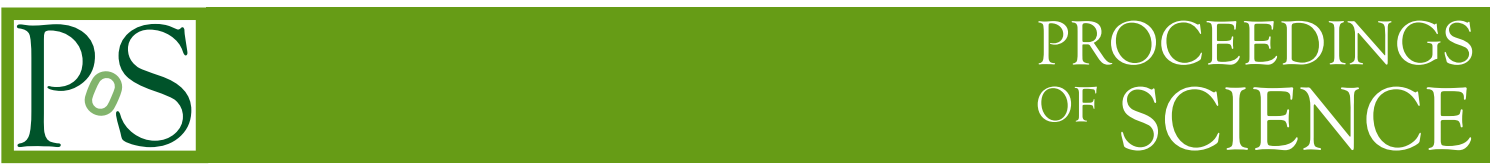

\title{
Omega Baryon Interactions with Lattice QCD
}

\author{
Joseph Wasem ${ }^{* \dagger}$ \\ Lawrence Livermore National Laboratory, L-414, 7000 East Ave., Livermore, CA 94550, USA \\ E-mail: wasem2@llnl.gov \\ LLNL-JRNL-522761
}

We discuss the interactions of the two- $\Omega^{-}$baryon system in multiple spin channels with lattice QCD. Lattice QCD is the only known technique for calculating low-energy hadronic observables directly from the underlying theory of QCD. In addition to being an interesting hyperonic system in its own right, the two- $\Omega^{-}$system also provides an ideal laboratory for exploring the interactions of multi-baryon systems with minimal dependence on light quark masses. Previous model calculations of the two- $\Omega^{-}$system have obtained conflicting results, which can be resolved by lattice QCD. The lattice calculations are performed using two different volumes with $L \sim 2.5$ and $3.9 \mathrm{fm}$ at $m_{\pi} \sim 390 \mathrm{MeV}$ with a lattice spacing of $a_{s} \sim 0.123 \mathrm{fm}$. Using multiple interpolating operators from a non-displaced source, we present scattering information for two ground state $\Omega^{-}$ baryons in both the $\mathrm{S}=0$ and $\mathrm{S}=2$ channels. For $\mathrm{S}=0, k \cot \delta$ is extracted at two volumes, which leads to an extrapolated scattering length of $a_{S=0}^{\Omega \Omega}=0.16 \pm 0.22 \mathrm{fm}$, indicating a weakly repulsive interaction. Additionally, for $\mathrm{S}=2$, two separate highly repulsive states are observed.

The 30th International Symposium on Lattice Field Theory

June 24 - 29, 2012

Cairns, Australia

\footnotetext{
* Speaker.

${ }^{\dagger}$ Work done in collaboration with Michael I. Buchoff and Thomas C. Luu.
} 
Lattice QCD calculations have advanced to the point that scattering phenomena for multihadron systems can be reliably calculated from first principles. Calculations performed with analysis of two or more hadrons in a finite volume allow for phase shifts and potential bound states to be studied non-pertubatively[1,2]. In this proceeding, we discuss the $\Omega^{-} \Omega^{-}$system, which is poorly understood experimentally due to the large mass and relatively short lifetime of the $\Omega^{-}$. Lattice QCD calculations can predict phenomena in these systems and pinpoint signals for heavy ion scattering experiments, such as STAR or ALICE. The $\Omega \Omega$ system has not received as much theoretical attention as its lighter hyperon counterparts, however within the last decade this system was studied in the context of the chiral quark model[3], where it was found to prefer a bound ground state with a binding energy of $116 \mathrm{MeV}$. A conflicting analysis[4] using the quark dislocation model finds the system to be weakly repulsive. A model-independent lattice QCD calculation can address this debate[5].

An attractive aspect of studying the $\Omega \Omega$ system on the lattice is the fact that the system is believed to primarily depend on the physical strange quark mass as opposed to the unphysically large light quark masses with which these lattice calculations are performed[6], implying a calculation at the physical point should rely less on chiral extrapolations. Also, calculations involving only the heavier strange quark are less computationally expensive and have better signal-to-noise. For these reasons, the multi- $\Omega^{-}$system is the ideal laboratory for understanding nuclear interactions (including tensor forces and s-wave three-baryon forces[7, 8]) directly from the lattice.

Lattice calculations are performed in Euclidean space, where the usual LSZ formalism only holds at kinematic threshold[9]. As a result, scattering information has to be extracted by analyzing energy shifts of hadrons in finite volume. For two hadrons $(A$ and $B)$ in a finite volume, the interaction momenta $k$ at a given volume $\left(V=L^{3}\right)$ can be related to the two-particle interaction energy $\Delta E$ and the scattering phase shifts $\delta(k)$ by $[1,2]$

$$
\begin{aligned}
E & =\sqrt{k^{2}+m_{A}^{2}}+\sqrt{k^{2}+m_{B}^{2}}=\Delta E+m_{A}+m_{B} \\
k \cot \delta(k) & =\frac{1}{\pi L} S\left(\left(\frac{k L}{2 \pi}\right)^{2}\right)=-\frac{1}{a}+\frac{1}{2} r k^{2}+\ldots \\
S(\eta) & =\sum_{\mathbf{j} \neq \mathbf{0}}^{|\mathbf{j}|<\Lambda} \frac{1}{|\mathbf{j}|^{2}-\eta}-4 \pi \Lambda
\end{aligned}
$$

where the value of $k \cot \delta(k)$ extracted then has the normal effective range expansion.

The discretized lattice calculations with periodic boundary conditions also break the continuum $O(3)$ rotational symmetry to an octahedral subgroup. The spin-3/2 $\Omega^{-}$fermionic modes map on to the lattice irrep $H^{+}(S=3 / 2)$. In this irrep, the interpolating operator overlapping the $\Omega^{-}$ baryon is given by[10]

$$
\bar{\Omega}_{\alpha \beta \gamma}=\varepsilon_{a b c} \bar{s}_{\alpha}^{a} \bar{s}_{\beta}^{b} \bar{s}_{\gamma}^{c},
$$

where $a, b$, and $c$ are color indices and $\alpha, \beta$, and $\gamma$ are spinor indices. Linear combinations of $\bar{\Omega}_{\alpha \beta \gamma}$ will produce $\Omega^{-}$interpolating operators in the correct irreducible representations of the octahedral group. For non-displaced sources, and following Ref. [10], there are two embeddings of the $\Omega^{-}$particle in the $H^{+}$irrep. In the non-relativistic limit, the first embedding maps onto the upper two spinor components in the Dirac-Pauli basis, while the second maps onto the lower 
two components. As such, one expects larger overlap with ground state systems when dealing with the first embedding. The s-wave states of the two $\Omega^{-}$system can be formed from a tensor product of the ground state lattice irreps, and by forcing the system of two $\mathrm{H}^{+}$baryons to be in a relative s-wave the angular momentum of the resulting state will be entirely determined by the spin combinations allowed. With the additional condition for an anti-symmetric wavefunction the two $\Omega^{-}$baryons in an s-wave state can only have two non-trivial spin channels: $S=0$ (the $A_{1}^{+}$irrep) and $S=2$ (the $E^{+}$and $T_{2}^{+}$irreps).

Due to the three degenerate valance quarks, the $\Omega^{-}$interpolating operator has the property that exchanging two quarks leads to both a minus sign from permuting Grassman number and a minus sign from exchanging indices in the epsilon tensor, which cancel, giving all Wick contractions the same relative sign. It can be shown using this property that all contractions of the two- $\Omega^{-}$fall into two distinct forms: 'direct' and 'exchange', as shown in Fig. 1. This drastically reduces the computational cost due to matrix multiplication, decreasing the $6 !=720$ possible contractions of two- $\Omega$ system by an order of magnitude.

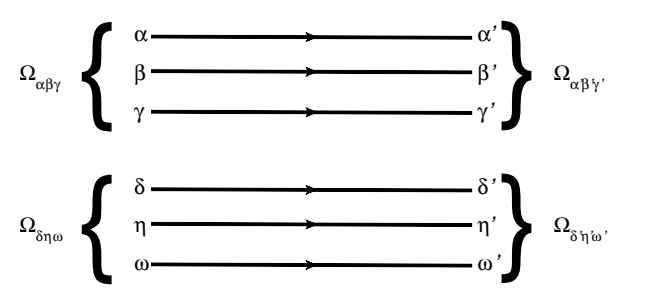

(a)

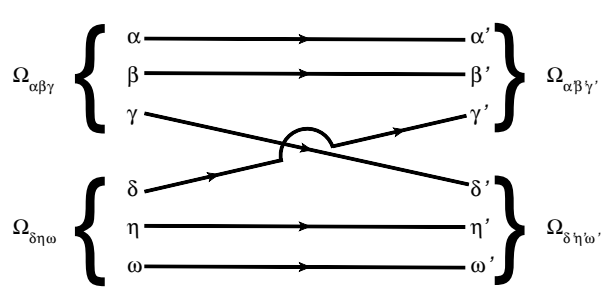

(b)

Figure 1: Quark contractions types used in the calculation including the (a) direct contributions and the (b) exchange contributions.

For each correlation function, different source/sink smearing combinations are put through a matrix-Prony algorithm as detailed in Ref. [11], which partially removes excited state contamination. Further enhancement of the ground state is accomplished by averaging different combinations of embeddings. We also suppress excited states by projecting the momentum of individual particle sinks to zero independently, which will remove excited states with nonzero back-to-back momentum[12]. A fully correlated $\chi^{2}$ minimizing fit is then performed in the plateau region to extract the ground state energy with errors.

Using Chroma[13], the configurations used were generated on uBGL while the propagator inversions and contractions were performed on the Edge cluster with the QUDA GPU library[14], both at LLNL. The configurations were anisotropic Wilson lattices using the tuning parameters defined in Ref. [15]. The ensembles used were $20^{3} \times 256(L \sim 2.5 \mathrm{fm})$ and $32^{3} \times 256(L \sim 3.9 \mathrm{fm})$ with $m_{\pi} \approx 390 \mathrm{MeV}, a_{s} \sim .1227 \mathrm{fm}$, and $a_{s} / a_{t} \sim 3.5$.

The three lowest embedding combinations of the $H^{+}$irrep have significant overlap with the ground state $\Omega^{-}$, and the effective mass plot for the sum of the three lowest embedding combinations is shown in Fig. 2 for both the $20^{3} \times 256$ and the $32^{3} \times 256$ lattices. The lowest embedding combinations for the $S=0$ system result in the effective mass plots in Fig. 3, with the corresponding energy shift of the two baryon system from ground state plotted in Fig. 4. The fit results for each data set are shown in Table 1. 


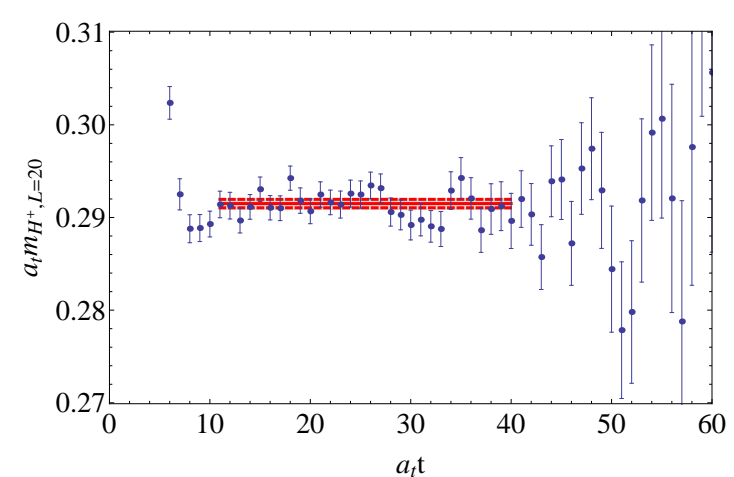

(a)

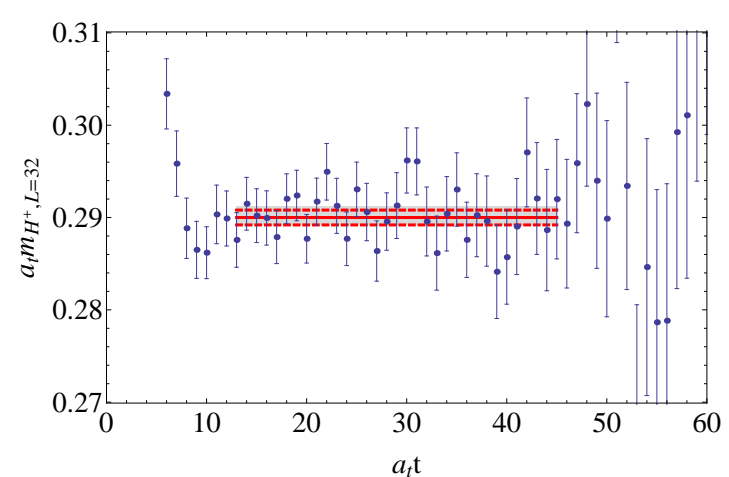

(b)

Figure 2: Effective mass plots for the $H^{+}\left(\mathrm{S}=\frac{3}{2}\right) \Omega^{-}$baryon calculated using (a) $20^{3} \times 256$ and (b) $32^{3} \times 256$ lattices.

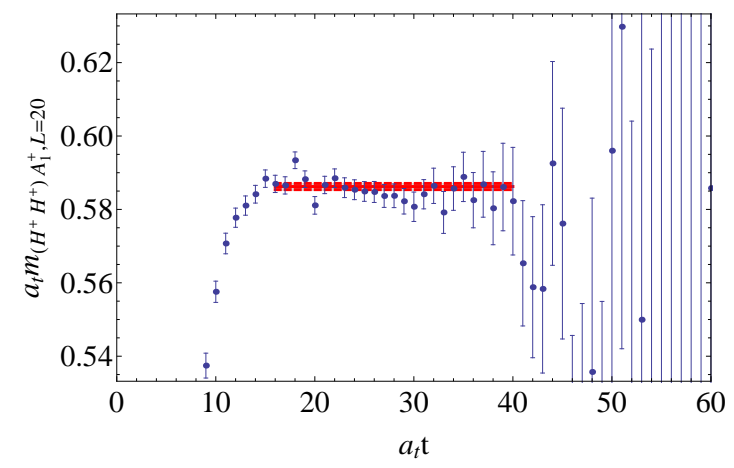

(a)

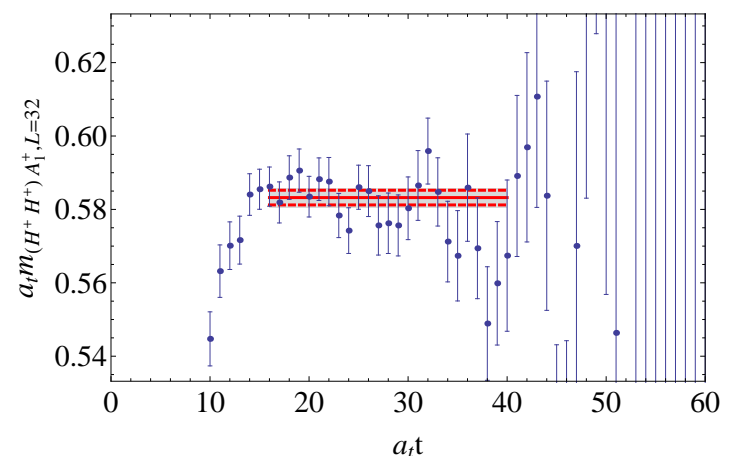

(b)

Figure 3: Effective mass plots for the $A_{1}^{+}$( $\mathrm{S}=0$ ) two $\Omega^{-}$system calculated using (a) $20^{3} \times 256$ and (b) $32^{3} \times 256$ lattices.

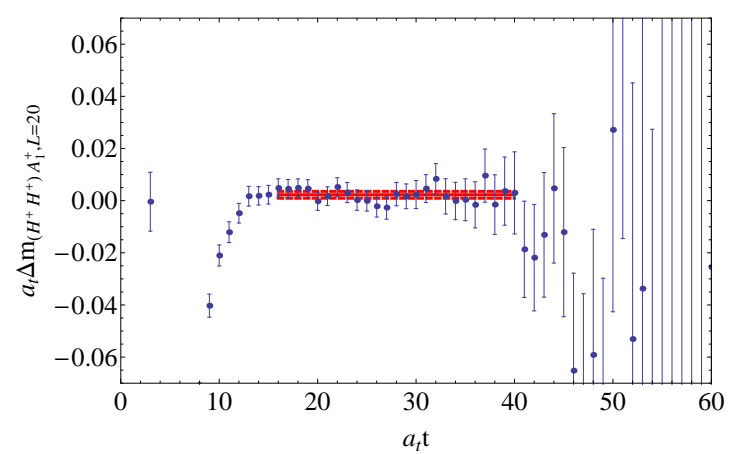

(a)

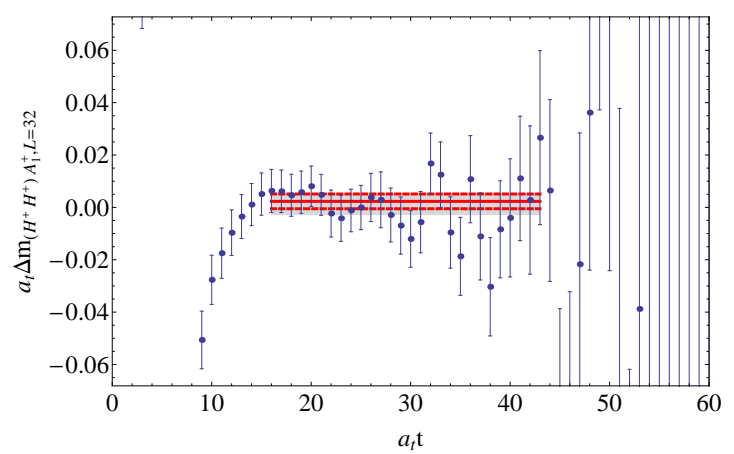

(b)

Figure 4: Effective mass plots for $\Delta E$ for the $A_{1}^{+}$( $\mathrm{S}=0$ ) two $\Omega^{-}$system calculated using (a) $20^{3} \times 256$ and (b) $32^{3} \times 256$ lattices. 


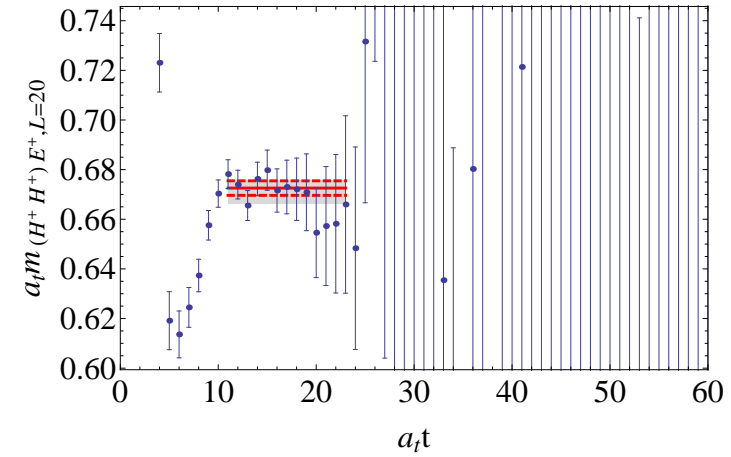

(a)

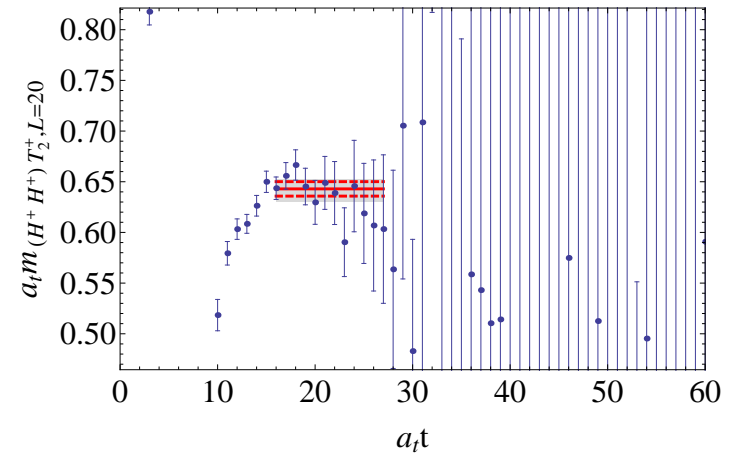

(b)

Figure 5: Effective mass plots of the (a) $E^{+}$and (b) $T_{2}^{+}$irreps (the $S=2$ two $\Omega^{-}$system) calculated using the $20^{3} \times 256$ lattice.

The effective mass plots for the $S=2$ states are shown in Fig. 5. One can see that the two $S=2$ irreps achieve statistically separate lowest energy states, despite coupling to states with the same set of quantum numbers, indicating that at least one (and possibly both) are failing to achieve the correct ground state of the $S=2$ two $\Omega^{-}$system. In both cases, the states achieved are at a significantly higher energy level than for the $S=0$ case, implying a much more repulsive channel, as expected from Pauli exclusion arguments.

Table 1: Fit values and Energy Shifts for the $\Omega^{-}$systems (in dimensionless units, $a_{t} E$ ).

\begin{tabular}{c|c|ccccccc} 
Irrep & Lattice Size & $a_{t} E$ & $\sigma_{E, \text { stat. }}$ & $\sigma_{E, \text { sys. }}$ & $\chi^{2} /$ dof & $\mathrm{Q}$ & $a_{t} \Delta E$ & $\sigma_{\Delta E, \text { stat. }}$ \\
\hline$H^{+}$ & $20^{3} \times 256$ & 0.291501 & 0.000457 & ${ }_{-0.000268}^{+0.000099}$ & 1.003 & 0.460 & & \\
& $32^{3} \times 256$ & 0.290001 & 0.000804 & ${ }_{-0.000418}^{+0.000001}$ & 0.850 & 0.708 & & \\
\hline$A_{1}^{+}$ & $20^{3} \times 256$ & 0.586235 & 0.000843 & ${ }_{-0.000091}^{+0.0000348}$ & 1.105 & 0.327 & 0.00323 & 0.00124 \\
& $32^{3} \times 256$ & 0.583224 & 0.002002 & ${ }_{-0.000577}^{+0.000680}$ & 1.086 & 0.350 & 0.00322 & 0.00257 \\
\hline$T_{2}^{+}$ & $20^{3} \times 256$ & 0.642961 & 0.007136 & ${ }_{-0.002502}^{+0.0005120}$ & 0.925 & 0.514 & 0.05996 & 0.00719 \\
$E^{+}$ & $20^{3} \times 256$ & 0.67256 & 0.00293 & ${ }_{-0.00132}^{+0.00013}$ & 0.500 & 0.916 & 0.08956 & 0.00307
\end{tabular}

In the $S=0$ two $\Omega^{-}$system, the data from two different volumes in Table 1 will allow for two applications of Eq. 1 and, in combination with the effective range expansion, an extraction of the scattering length $a$. In principle the range parameter $r$ will also be extracted, however it will be contaminated by higher order terms and is unreliable. For the $S=0$ system the results are shown in Fig. 6(a) along with the systematic and statistical errors. A distribution of the parameter $a$ is generated and shown in Fig. 6(b), with a resulting scattering length in the $S=0$ channel of

$$
a_{S=0}^{\Omega \Omega}=0.16 \pm 0.22 \mathrm{fm} .
$$

Note that the distribution fit to the data is that of a Lorentz distribution, where use of a normal distribution would result in the quotation of too large an uncertainty.

From Fig. 6(a) one can see that the central value of $k^{2}$ observed does not change appreciably between the two different volumes, and in Fig. 6(b) the distribution of the extracted scattering 


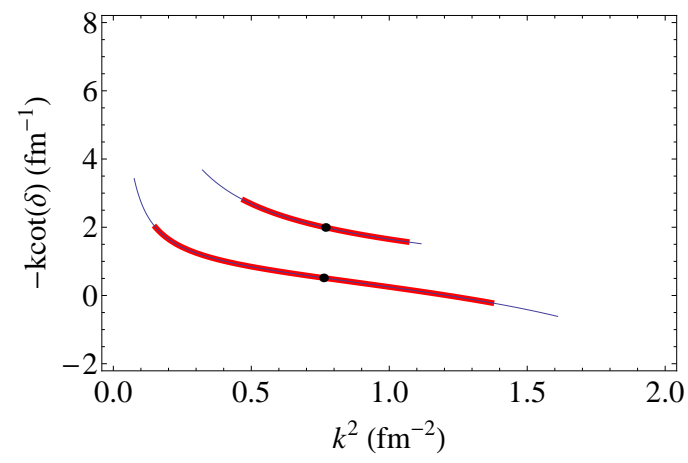

(a)

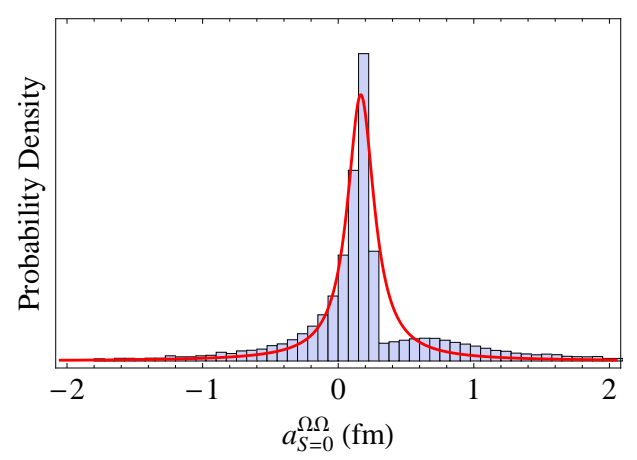

(b)

Figure 6: Plot of (a) $k \cot \delta$ (with central value, statistical error (thick line), and statistical plus systematic error (thin line)) and (b) the distribution of scattering lengths $a$ for the $S=0$ two $\Omega^{-}$system with a Lorentz distribution.

length is strongly peaked at very small values, indicating a very weakly repulsive system. Indeed, assuming natural sizes for the higher order range parameters the Lorentz distribution for the scattering length provides a $79.5 \%$ chance that the system is repulsive and a $20.5 \%$ chance that it is attractive. Additionally, the $\Delta E$ values in Table 1 are positive (repulsive) and small for both $20^{3} \times 256$ and $32^{3} \times 256$ lattices. Thus, from our current calculations, we find evidence that the system is consistent with the weakly repulsive scenario in Ref. [4] and inconsistent with the deeply bound state found in Ref. [3]. Ultimately, more calculations are required to acquire a full systematic error budget, but for the $\Omega \Omega$ system these are expected to be small due to weak light quark dependence.

These results provide an interesting complement to previous studies[12, 16, 17, 18] of hyperon interactions, where many of the interactions have been found to be attractive and contain bound states at $m_{\pi} \sim 390 \mathrm{MeV}$. The difference between the evidence for other bound hyperon states and the conclusion in this work of a weakly repulsive $\Omega \Omega$ state may simply reflect a much stronger influence of light-quark dynamics in the valence sector of the $\Lambda \Lambda$ and $\Xi \Xi$ systems. Further studies at different pion masses approaching the physical point are needed to gain a better understanding of the similarities and differences of each of these systems.

This work was performed under the auspices of the U.S. DOE by LLNL under Contract No. DE-AC52-07NA27344 and the UNEDF SciDAC Grant No. DE-FC02-07ER41457, and was partially supported by LLNL LDRD 10-ERD-033.

\section{References}

[1] M. Luscher, Commun. Math. Phys. 105, 153 (1986).

[2] M. Luscher, Nucl.Phys. B354, 531 (1991).

[3] Z. Y. Zhang, Y. W. Yu, C. R. Ching, T. H. Ho, and Z.-D. Lu, Phys. Rev. C61, 065204 (2000).

[4] F. Wang, J.-l. Ping, G.-h. Wu, L.-j. Teng, and J. T. Goldman, Phys. Rev. C51, 3411 (1995).

[5] M. I. Buchoff, T. C. Luu, and J. Wasem, (2012), arXiv:1201.3596 [hep-lat].

[6] J. Bulava et al., Phys. Rev. D82, 014507 (2010), arXiv:1004.5072 [hep-lat]. 
[7] S. R. Beane, W. Detmold, T. C. Luu, K. Orginos, A. Parreno, et al., Phys.Rev. D80, (2009), arXiv:0905.0466 [hep-lat].

[8] T. Yamazaki, Y. Kuramashi, A. Ukawa, and f. t. P.-C. Collaboration, Phys.Rev. D81, 111504 (2010), arXiv:0912.1383 [hep-lat].

[9] L. Maiani and M. Testa, Phys. Lett. B245, 585 (1990).

[10] S. Basak et al., Lattice Hadron Physics Collaboration (LHPC), Phys. Rev. D72, 074501 (2005), arXiv:hep-lat/0508018.

[11] S. R. Beane et al., Phys. Rev. D79, 114502 (2009), arXiv:0903.2990 [hep-lat].

[12] S. R. Beane et al. (NPLQCD), Phys. Rev. D81 054505 (2010), arXiv:0912.4243 [hep-lat].

[13] R. G. Edwards and B. Joo, Nucl. Phys. Proc. Suppl. 140, 832 (2005), arXiv:hep-lat/0409003.

[14] M. A. Clark, R. Babich, K. Barros, R. C. Brower, and C. Rebbi, Comput. Phys. Commun. 181, 1517 (2010), arXiv:0911.3191 [hep-lat].

[15] H.-W. Lin et al. (Hadron Spectrum Collaboration), Phys. Rev. D79, 034502 (2009), arXiv:0810.3588 [hep-lat].

[16] S. R. Beane et al. (NPLQCD), (2010), arXiv:1012.3812 [hep-lat].

[17] T. Inoue et al. (HAL QCD), Phys. Rev. Lett. 106, 162002 (2011), arXiv:1012.5928 [hep-lat].

[18] S. R. Beane et al. (NPLQCD), (2011), arXiv:1109.2889 [hep-lat]. 